\title{
This stone (may?) too shall pass
}

\author{
Jennifer Bjazevic, BHSc, MSc, MD, FRCSC
}

Division of Urology, Western University, London, ON, Canada

Cite as: Bjazevic J. This stone (may?) too shall pass. Can Urol Assoc J 2021;15(12):412. http://dx.doi.org/10.5489/cuaj.7678

See related article on page 407

I $\mathrm{n}$ this issue of CUAJ, González-Padilla et al, in a retrospective review, examined the impact of surgical wait times on the rate of negative ureteroscopy (URS) for the treatment of ureteric calculi $<10 \mathrm{~mm}$ in size. ${ }^{1}$ The study reports an overall $18.7 \%$ rate of negative URS and found that larger stone size, the presence of a ureteric stent, and radio-opaque calculi all decreased the likelihood of negative ureteroscopy. ${ }^{1}$ The median surgical wait time reported in the study was 74 days, which did not include the average 4-6-week waiting period for patients to receive a urological consultation after first presenting with symptoms of renal colic. ${ }^{1}$ Overall, the authors concluded that longer surgical wait times were significantly associated with a higher rate of negative URS. ${ }^{1}$

It is interesting to note that the authors report a slightly higher rate of negative URS (18.7\%) than what has previously been reported in the literature, where rates have ranged from $3.9-13.7 \% .^{2}$ This is possibly due to the exclusion of stones $>10 \mathrm{~mm}$ in size and the longer surgical wait times in this series. ${ }^{3}$ The findings of this study are consistent with previous reports, which have also found smaller stone size and radio-lucent stones to be associated with higher rates of negative URS. ${ }^{4,5}$ Additional factors, including female gender and stone location in the distal ureter, have also previously been found to be predictive of negative URS. ${ }^{3,5}$

The authors should be commended on publishing the first report specifically examining the effect of surgical wait times on the incidence of negative URS. While URS is a very safe and commonly performed procedure, it is not without risks; a global study performed by the Clinical Research Office of the Endourological Society (CROES) has previously demonstrated an overall $3.5 \%$ complication rate with URS. ${ }^{6}$ In addition to operative and anesthetic risks, negative URS also results in unnecessary healthcare expenditures and social costs, including sick leave. Consequently, diligent efforts should be made to avoid the occurrence of negative URS whenever possible.

Obtaining repeat imaging prior to surgery can significantly reduce the rate of negative URS but comes at the cost

of increased radiation exposure to patients, and these risks need to be balanced. Patients with radio-opaque stones previously visible on $\mathrm{X}$-ray should undergo a repeat $\mathrm{X}$-ray prior to URS to ensure persistence of their stone. Repeat imaging with low-dose non-contrast computed tomography (CT) scan should be considered for patients with radio-lucent stones or those not clearly visible on X-ray prior to URS - especially for patients at high risk for negative URS.

To maximize the utility of repeat imaging, these exams should be performed as close to the time of surgery as possible. The importance of this was well-demonstrated in this current series, where patients with radio-opaque stones routinely had a repeat X-ray the day before surgery, but despite this, still demonstrated a relatively high rate of negative URS (14.9\%).

The individualization of preoperative imaging strategies and judicious use of low-dose CT scans can help to mitigate patient radiation exposure while minimizing the rates of negative URS. In addition, informed discussion with the patient regarding the risks of repeated imaging exams and negative URS, as well as the use of a shared decision-making model, can help guide clinical practice.

Competing interests: The author does not report any competing personal or financial interests related to this work.

\section{References}

1. Gonzalez-Padilla D, Gonzalez-Diaz A, Pena-Vallejo $\mathrm{H}$ et al. Long surgical waiting list times are associated with an increased rate of negative ureteroscopies. Can Urol Assoc J 2021;15:407-11. https://doi.org/10.5489/cuaj.7199

2. Rice P, Prattley S, Somani BK. 'Negative ureteroscopy' for stone disease: Evidence from a systematic review. Curr Urol Rep. 2019;20:13-9. https://doi.org/10.1007/s11934-019-0878-4

3. Sahin $M$, Sen V, Irer B, et al. Can negative ureteroscopy be predicted in ureteral stone treatment? Can Urol Assoc J 2019;14:E209-13. hitps://doi.org/10.5489/cuaj.6026

4. Katafigiotis I, Sabler IM, Heifetz EM, et al. "Stoneless" or negative ureteroscopy: A reality in the endourologic routine or avoidable source of frustration? Estimating the risk factors for negative ureteroscopy. J Endourol 2018;32:825-30. https://doi.org/10.1089/end.2018.0291

5. Kreshover JE, Dickstein RJ, Rowe C, et al. Predictors for negative ureteroscopy in the management of upper urinary tract stone disease. Urology 2011;78:748-52. https://doi.org/10.1016/i.urology.2011.03.008

6. Somani BK, Giusti G, Sun Y, el al. Complications associated with ureteroscopy (URS) related to treatment of urolithiasis: The Clinical Office of Endourological Society URS Global Study. World J Urol 2017;35:67581. https://doi.org/10.1007/s00345-016-1909-0

Correspondence: Dr. Jennifer Bjazevic, Division of Urology, Western University, London, ON, Canada; Jennifer.bjazevic@sihc.london.on.ca 